\title{
Symmetric Time-Reversible Flows with a Strange Attractor
}

\author{
J. C. Sprott \\ Department of Physics, University of Wisconsin, \\ Madison, WI 53706, USA \\ sprott@physics.wisc.edu
}

Received January 12, 2015

\begin{abstract}
A symmetric chaotic flow is time-reversible if the equations governing the flow are unchanged under the transformation $t \rightarrow-t$ except for a change in sign of one or more of the state space variables. The most obvious solution is symmetric and the same in both forward and reversed time and thus cannot be dissipative. However, it is possible for the symmetry of the solution to be broken, resulting in an attractor in forward time and a symmetric repellor in reversed time. This paper describes the simplest three-dimensional examples of such systems with polynomial nonlinearities and a strange (chaotic) attractor. Some of these systems have the unusual property of allowing the strange attractor to coexist with a set of nested symmetric invariant tori.
\end{abstract}

Keywords: Chaos; symmetry; attractor; time-reversal invariance.

\section{Introduction}

At the microscopic level, the laws of physics are time-reversible. To deal with such systems, Hamiltonian mechanics was developed long ago, and it works well even when the solutions are chaotic as, for example, in the three-body gravitational problem. However, Hamiltonian systems do not allow the kind of strange attractors that are evident in most natural systems that are driven out of equilibrium by some source of energy that is dissipated as heat and leads to an increase in entropy according to the Second Law of Thermodynamics. In the dynamical equations, the energy source usually takes the form of positive feedback, and the dissipation is in some kind of frictional damping. Chaotic systems generally have strange attractors in the reversed-time direction if the parameters are altered to reverse the sign of the damping and feedback terms, but the interest here is in relatively rare examples that have strange attractors in both forward and reversed time for the same parameters.
Thus it is interesting to consider symmetric continuous-time dynamical systems that are timereversible and that also have strange attractors, and in particular, to find the simplest examples of such systems, which are common in physical problems, but are rarely studied mathematically. In that spirit, the present paper considers threedimensional autonomous flows with quadratic nonlinearities, the most general form of which is

$$
\begin{aligned}
\dot{x}= & a_{1}+a_{2} x+a_{3} y+a_{4} z+a_{5} x^{2}+a_{6} y^{2} \\
& +a_{7} z^{2}+a_{8} x y+a_{9} x z+a_{10} y z \\
\dot{y}= & a_{11}+a_{12} x+a_{13} y+a_{14} z+a_{15} x^{2} \\
& +a_{16} y^{2}+a_{17} z^{2}+a_{18} x y+a_{19} x z+a_{20} y z \\
\dot{z}= & a_{21}+a_{22} x+a_{23} y+a_{24} z+a_{25} x^{2} \\
& +a_{26} y^{2}+a_{27} z^{2}+a_{28} x y+a_{29} x z+a_{30} y z .
\end{aligned}
$$

The simplest type of time-reversal invariance would be $(x, y, z, t) \rightarrow(x, y, z,-t)$, which reverses 
the sign of all 30 of the coefficients ( $a_{1}$ through $a_{30}$ ) in Eq. (1) and hence leads to the trivial case $\dot{x}=$ $\dot{y}=\dot{z}=0$. Of more interest are cases in which time is reversed as well as the sign of three, two, or one of the state space variables. These three cases will be considered in turn in the following sections.

\section{Inversion Invariant Systems}

For systems that are invariant under the transformation $(x, y, z, t) \rightarrow(-x,-y,-z,-t)$, only the constant and quadratic terms in Eq. (1) survive, resulting in a system of the form

$$
\begin{aligned}
\dot{x}= & a_{1}+a_{2} x^{2}+a_{3} y^{2}+a_{4} z^{2} \\
& +a_{5} x y+a_{6} x z+a_{7} y z \\
\dot{y}= & a_{8}+a_{9} x^{2}+a_{10} y^{2}+a_{11} z^{2} \\
& +a_{12} x y+a_{13} x z+a_{14} y z \\
\dot{z}= & a_{15}+a_{16} x^{2}+a_{17} y^{2}+a_{18} z^{2} \\
& +a_{19} x y+a_{20} x z+a_{21} y z .
\end{aligned}
$$

Systems of this type were recently explored [Li \& Sprott, 2014], and the simplest chaotic example is given by

$$
\begin{aligned}
& \dot{x}=1+y z \\
& \dot{y}=-x z \\
& \dot{z}=y^{2}+a y z
\end{aligned}
$$

which for $a=2$ has a symmetric attractor/repellor pair as shown in Fig. 1. The dissipation, or equivalently, the rate of state space contraction, is given by the negative trace of the Jacobian matrix timeaveraged along the orbit, $-\langle a y\rangle=2.2350$. The Lyapunov exponents are $(0.1520,0,-2.3870)$, and the Kaplan-Yorke dimension is 2.0637. When time is reversed, the attractor becomes a repellor, and the repellor becomes an attractor with identical characteristics, which is a general feature of timereversible systems with a strange attractor [Moran et al., 1987]. Of course, the Kaplan-Yorke dimension is not strictly defined for a repellor, but its dimension is identical to that of the corresponding attractor as a consequence of the symmetry. Such examples are well known in iterated maps [Hoover et al., 1996] but have been relatively little studied in ordinary differential equations.

In principle, the system in Eq. (3) could have conservative solutions, as one might expect for a

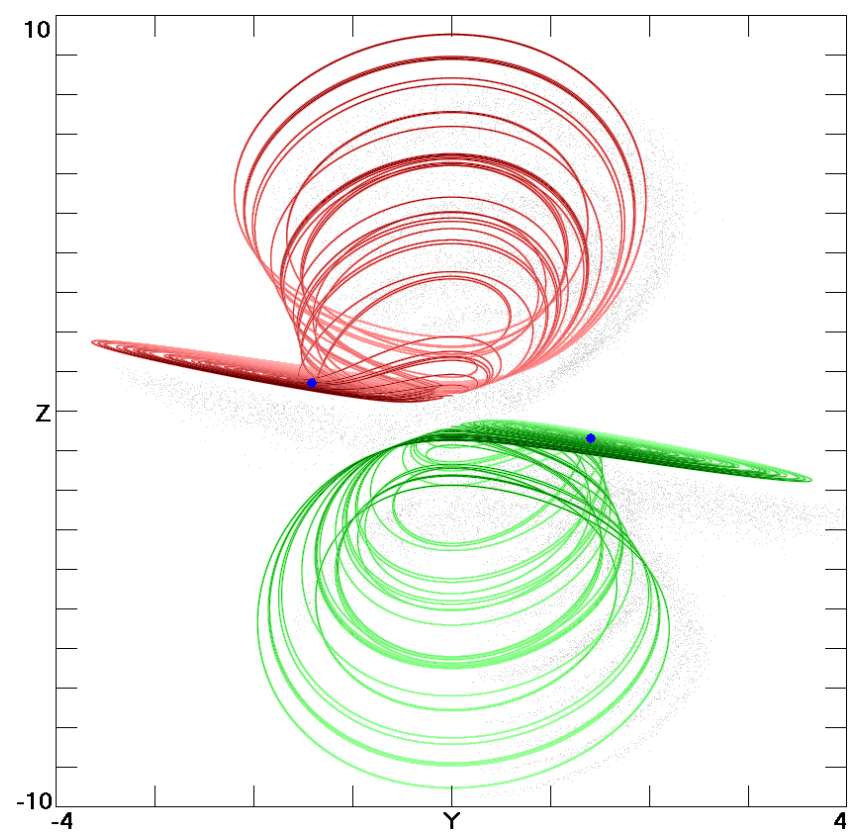

Fig. 1. Attractor (red) and repellor (green) from Eq. (3) with $a=2$ and initial conditions $(0,0,2)$. The two saddle points (eigenvalues: $-2.979261,0.075417 \pm 0.971434 i$ and $2.979261,-0.075417 \pm 0.971434 i)$ are shown as blue dots.

time-reversible system if the solution were symmetric about the $y=0$ axis so that the dissipation $-\langle a y\rangle$ would be exactly zero. Such solutions apparently do not occur for any value of the parameter $a$ and initial conditions.

Furthermore, there is a variant of Eq. (3) given by

$$
\begin{aligned}
& \dot{x}=1-y^{2} \\
& \dot{y}=-x z \\
& \dot{z}=y^{2}+a y z
\end{aligned}
$$

in which the $y z$ term in the $\dot{x}$ equation is replaced with $-y^{2}$, and it also has an attractor/repellor pair for $a=2.5$ as shown in Fig. 2 but no conservative solutions. The dissipation is given by $-\langle a y\rangle=1.9012$, and the basin of attraction is relatively small. The Lyapunov exponents are $(0.0739,0,-1.9751)$, and the Kaplan-Yorke dimension is 2.0374 .

These appear to be the only systems in the form of Eq. (2) with five or fewer terms and a strange attractor. Three-dimensional systems with five terms are desirable because they are completely governed by a single parameter since four of the coefficients can usually be set to \pm 1 by a linear rescaling of $x, y, z$, and $t$. Many similar examples 


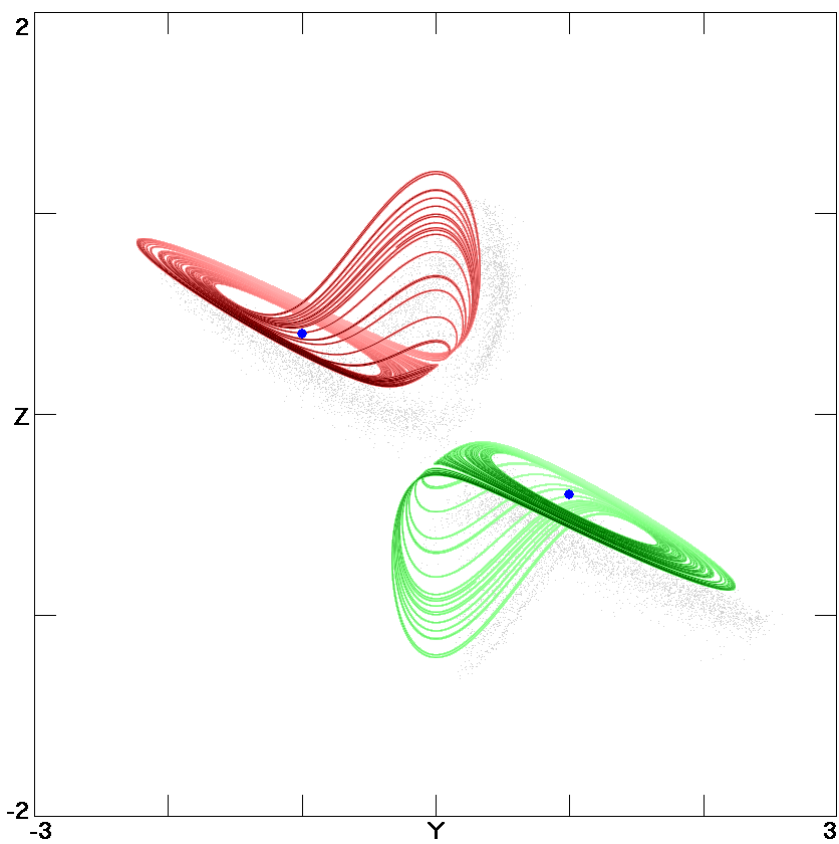

Fig. 2. Attractor (red) and repellor (green) from Eq. (4) with $a=2.5$ and initial conditions $(0,0,0.24)$. The two equilibrium points (eigenvalues: $-2.5, \pm 0.894427 i$ and 2.5, $\pm 0.894427 i)$ are shown as blue dots.

of systems with six terms exist but will not be further explored here in the spirit of identifying the simplest such systems.

\section{Rotation Invariant Systems}

Systems that are invariant under the transformation $(x, y, z, t) \rightarrow(x,-y,-z,-t)$ have a timereversed dynamic that is symmetric with the one for forward time but rotated by $180^{\circ}$, here taken about the $x$-axis without loss of generality. The most general such three-dimensional quadratic form is

$$
\begin{aligned}
\dot{x}= & a_{3} y+a_{4} z+a_{8} x y+a_{9} x z \\
\dot{y}= & a_{11}+a_{12} x+a_{15} x^{2}+a_{16} y^{2} \\
& +a_{17} z^{2}+a_{20} y z \\
\dot{z}= & a_{21}+a_{22} x+a_{25} x^{2}+a_{26} y^{2} \\
& +a_{27} z^{2}+a_{30} y z .
\end{aligned}
$$

An example of a system of this form has recently been described [Sprott, 2014] and is given by

$$
\begin{aligned}
& \dot{x}=y+2 x y+x z \\
& \dot{y}=1-2 x^{2}+y z \\
& \dot{z}=x-x^{2}-y^{2} .
\end{aligned}
$$

All orbits of this system are bounded, and it has no equilibrium points. It also has the unusual property of having a chaotic attractor/repellor pair and a set of nested symmetric invariant tori for the same values of the parameters. Initial conditions of $(2,0,0)$ give a strange attractor, and $(1,0,0)$ give an invariant torus. The rate of state space contraction, is given by $-2\langle y+z\rangle$, which has a value of 0.1035 for the strange attractor and $0 \pm 10^{-10}$ for the torus. For the strange attractor, the Lyapunov exponents are $(0.0540,0,-0.1575)$, and the Kaplan-Yorke dimension is 2.3429. The goal of this section is to identify and describe even simpler examples of such behavior.

The simplest example of a system in the form of Eq. (5) with a strange attractor was long ago identified [Sprott, 1994] (Case D) and is given (with a transformation of variables) by

$$
\begin{aligned}
& \dot{x}=y+z \\
& \dot{y}=-x \\
& \dot{z}=a x^{2}+y z .
\end{aligned}
$$

For $a=3$, it has a strange attractor/repellor pair as shown in Fig. 3 but apparently no conservative solutions for any value of $a$. The Lyapunov exponents are $(0.1027,0,-1.3198)$, and the KaplanYorke dimension is 2.0778 . The basin of attraction

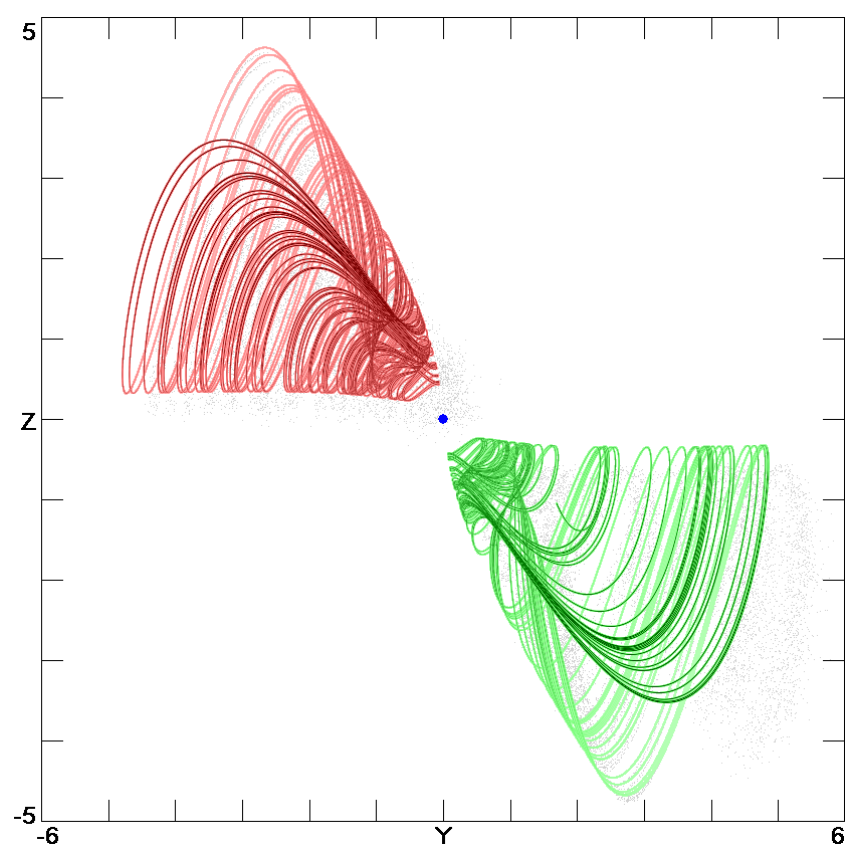

Fig. 3. Attractor (red) and repellor (green) from Eq. (7) with $a=3$ and initial conditions $(0,-0.23,1)$. The center (eigenvalues: $0, \pm i$ ) at the origin is shown as a blue dot. 
is relatively large, occupying about a third of the volume of the state space.

A simple system in the form of Eq. (5) with a conservative chaotic sea and coexisting invariant tori, but without a strange attractor, was also identified in the same paper (Case A) as given by

$$
\begin{aligned}
& \dot{x}=y \\
& \dot{y}=-x+y z \\
& \dot{z}=1-y^{2} .
\end{aligned}
$$

It was later pointed out [Hoover, 1995] that this system is a special case of the Nosé-Hoover thermostated oscillator [Nosé, 1991] with a spatially constant temperature. This system is also unusual since it has no equilibrium points.

A generalization of Eq. (8) with a nonuniform temperature gradient has recently been studied [Sprott et al., 2014] as given by

$$
\begin{aligned}
& \dot{x}=y \\
& \dot{y}=-x-y z \\
& \dot{z}=y^{2}-1-\epsilon \tanh (x) .
\end{aligned}
$$

For $\epsilon=0.42$, it has an attracting limit cycle coexisting with a pair of nested invariant tori, and for $\epsilon=0.38$ it has a multifractal strange attractor coexisting with a complicated set of invariant tori. For the strange attractor, the Lyapunov exponents are $(0.0019,0,-0.0020)$, and the Kaplan-Yorke dimension is 2.945 . The dissipation for the strange attractor is very small, approximately $1.2 \times 10^{-4}$, but decidedly nonzero.

A simplification of Eq. (9) with a somewhat nonphysical constant temperature gradient is given by

$$
\begin{aligned}
& \dot{x}=y \\
& \dot{y}=-x-y z \\
& \dot{z}=y^{2}-a-x .
\end{aligned}
$$

For $a=1.2$, this system has a strange attractor/repellor pair that coexists with a set of nested invariant tori, one of which is shown in Fig. 4. The strange attractor is produced using initial conditions $(0,0.2,1)$, and the torus is produced using initial conditions $(-0.5,0.75,0)$. Note that the strange attractor links the torus three times. The symmetric repellor is not shown, but in this plane it is identical to the attractor but upside down and intertwined with it. For the strange attractor, the Lyapunov

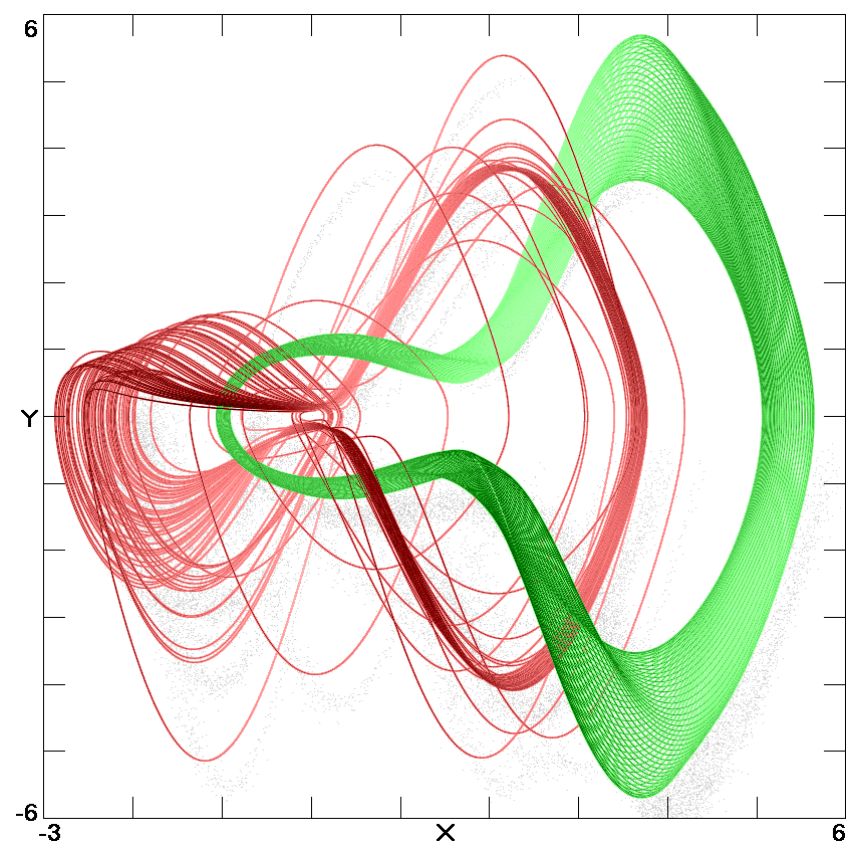

Fig. 4. Strange attractor (red) coexisting with an invariant torus (green) from Eq. (10) with $a=1.2$. The symmetric repellor is not shown. This system has no equilibrium points, and thus the strange attractor is hidden.

exponents are $(0.0472,0,-0.8631)$, and the KaplanYorke dimension is 2.0547. The basin of attraction is relatively large, occupying about half the volume of the state space. As for Eq. (6), this system has no equilibrium points, and thus the strange attractor is hidden in the sense that it cannot be found by choosing an initial condition on the unstable manifold in the neighborhood of an equilibrium point [Leonov et al., 2011; Leonov \& Kuznetsov, 2013].

Since the intermingling of the strange attractor, its symmetric repellor, and the set of nested tori is hard to visualize in Fig. 4, a cross-section of the state space for $z=0$ is shown in Fig. 5 for various initial values of $x$ with $y=z=0$. This plot is not called a "Poincaré section" because it shows orbits crossing the $z=0$ plane going in both directions. The nested tori (shown in black) are clearly seen. The repellor (shown in green) appears to intersect the strange attractor (shown in red) in some regions of space, but the dissipative and conservative regions are clearly separated and interlinked. The light blue background shows initial conditions that give conservative tori, and the yellow background indicates the basin of attraction of the strange attractor.

A system with one less term than Eq. (10) but with three rather than two nonlinearities is given 


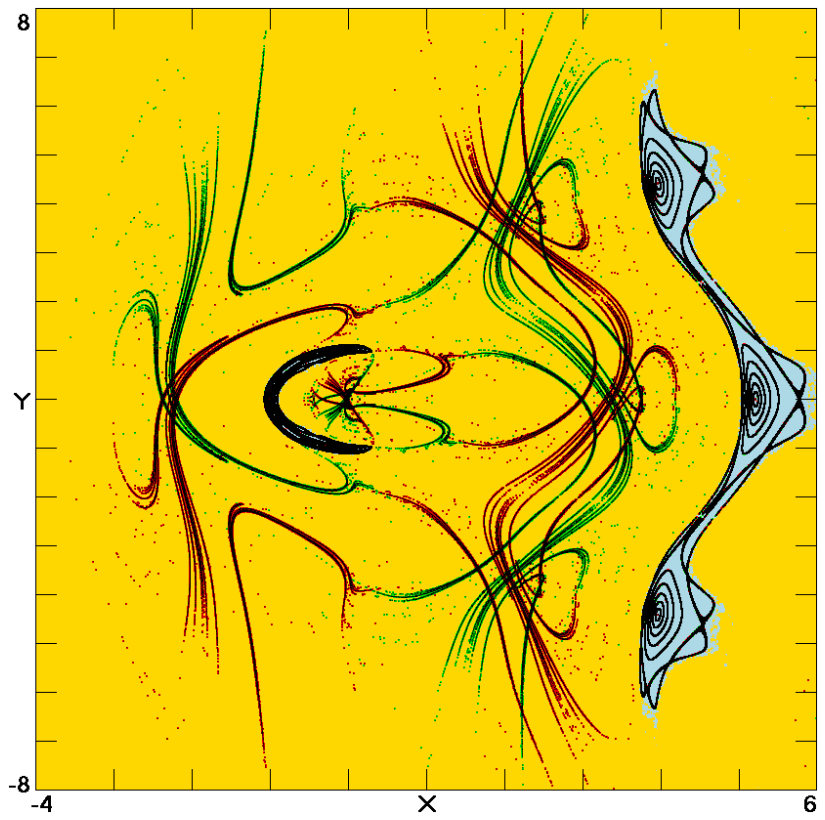

Fig. 5. Cross-section in the plane $z=0$ of the strange attractor (red), its symmetric repellor (green), and the coexisting invariant tori (black) from Eq. (10) with $a=1.2$. The light blue background shows initial conditions that give conservative tori, and the yellow background indicates the basin of attraction of the strange attractor.

by

$$
\dot{x}=z, \quad \dot{y}=x, \quad \dot{z}=a x^{2}-y^{2}-y z
$$

which is chaotic for $a=1.3$ with a strange attractor/repellor pair, but without coexisting invariant

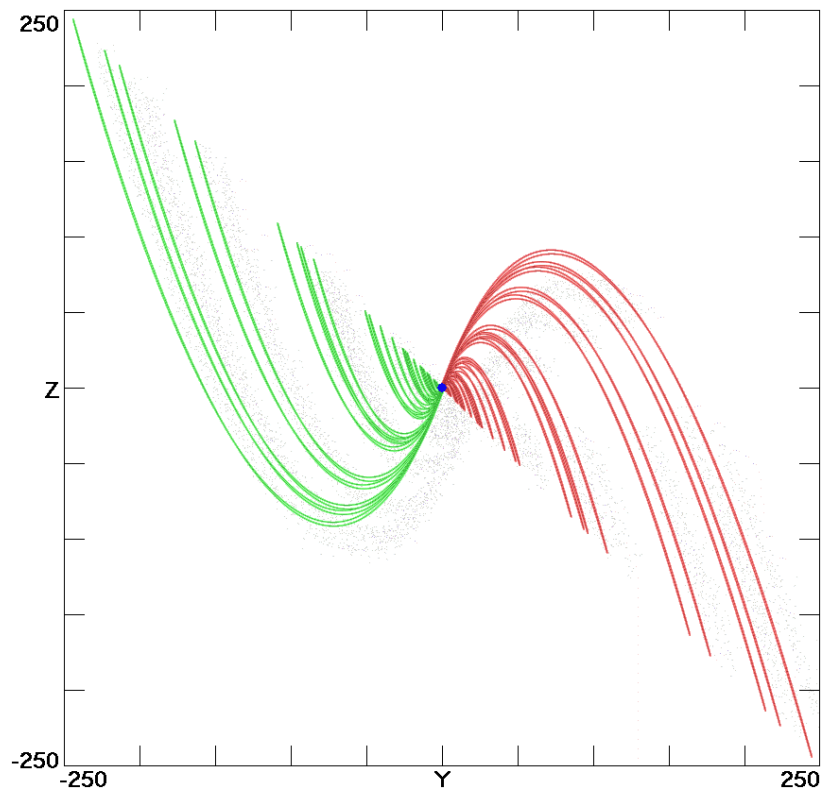

Fig. 6. Attractor (red) and repellor (green) from Eq. (11) with $a=1.3$ and initial conditions $(-0.6,0,0.2)$. The equilibrium (eigenvalues: $0,0,0)$ at the origin is shown as a blue dot. tori. The system can be written more compactly in jerk form as $\dddot{y}=a \dot{y}^{2}-y^{2}-y \ddot{y}$. The basin of attraction occupies about a third of the volume of the state space, and an initial condition of $(-0.6,0,0.2)$ is in the basin and close to the attractor. The equation is relatively stiff, and the attractor is unusual in the sense that the orbit spends a long time in the vicinity of the origin with occasional excursions far away as shown in Fig. 6 along with the symmetric repellor. The only equilibrium is at the origin, and it has eigenvalues that satisfy $\lambda^{3}=0$. The damping is very large but very brief and is given by $\langle y\rangle=14.3188$. The Lyapunov exponents are $(0.0359,0,-14.3547)$, and the KaplanYorke dimension is 2.0025 .

\section{Reflection Invariant Systems}

Systems that are invariant under the transformation $(x, y, z, t) \rightarrow(x, y,-z,-t)$ have a time-reversed dynamic that is symmetric with the one for forward time but reflected in a plane here taken as $z=0$ without loss of generality. The most general such three-dimensional quadratic form is

$$
\begin{aligned}
\dot{x}= & \left(a_{4}+a_{9} x+a_{10} y\right) z \\
\dot{y}= & \left(a_{14}+a_{19} x+a_{20} y\right) z \\
\dot{z}= & a_{21}+a_{22} x+a_{23} y+a_{25} x^{2} \\
& +a_{26} y^{2}+a_{27} z^{2}+a_{28} x y .
\end{aligned}
$$

However, there do not appear to be any chaotic solutions for Eq. (12).

On the other hand, when the 14 cubic terms that satisfy the symmetry conditions are added, chaotic solutions occur. One simple case is given by

$$
\begin{aligned}
& \dot{x}=-y z \\
& \dot{y}=\left(a x+y+z^{2}\right) z \\
& \dot{z}=x-x^{3} .
\end{aligned}
$$

For $a=2$, it has a strange attractor/repellor pair symmetric about the $z=0$ plane as shown in Fig. 7 . The Lyapunov exponents are $(0.0892,0,-1.2270)$, and the Kaplan-Yorke dimension is 2.0727. The basin of attraction is relatively small.

This system is unusual because it has two equilibrium points at $(-1,0, \pm \sqrt{2})$ with eigenvalues $(-2.090161,0.337974 \pm 2.301872 i)$, respectively, and three infinite lines of equilibrium points at $(0,0, z)$ 


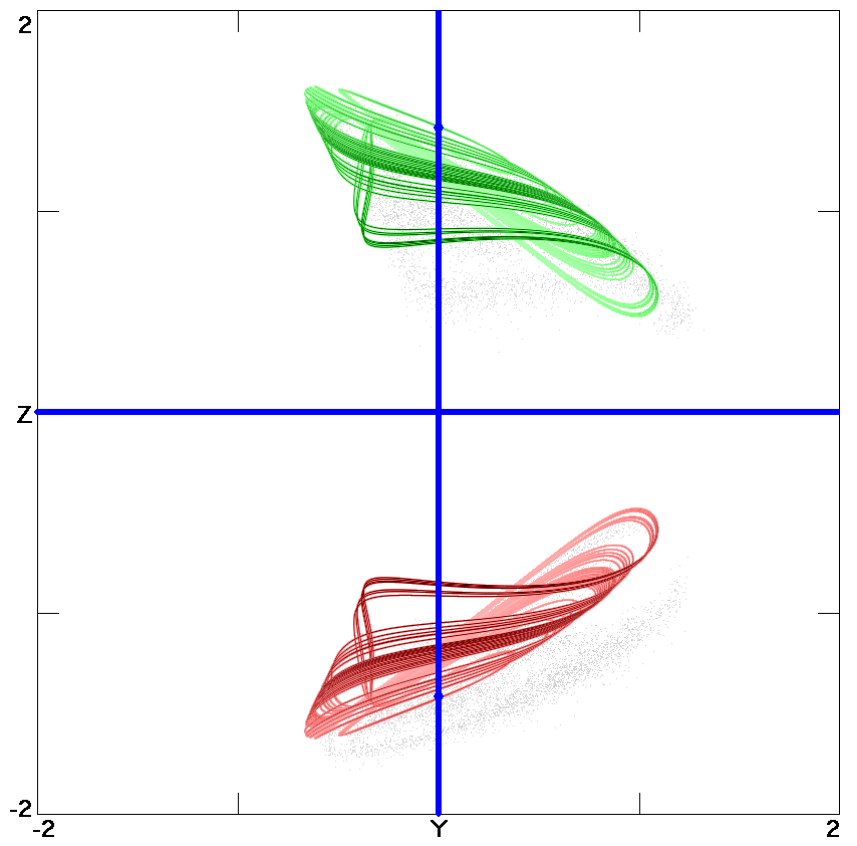

Fig. 7. Attractor (red) and repellor (green) from Eq. (13) with $a=2$ and initial conditions $(0,0,-0.94)$. The lines of equilibrium points are shown in blue.

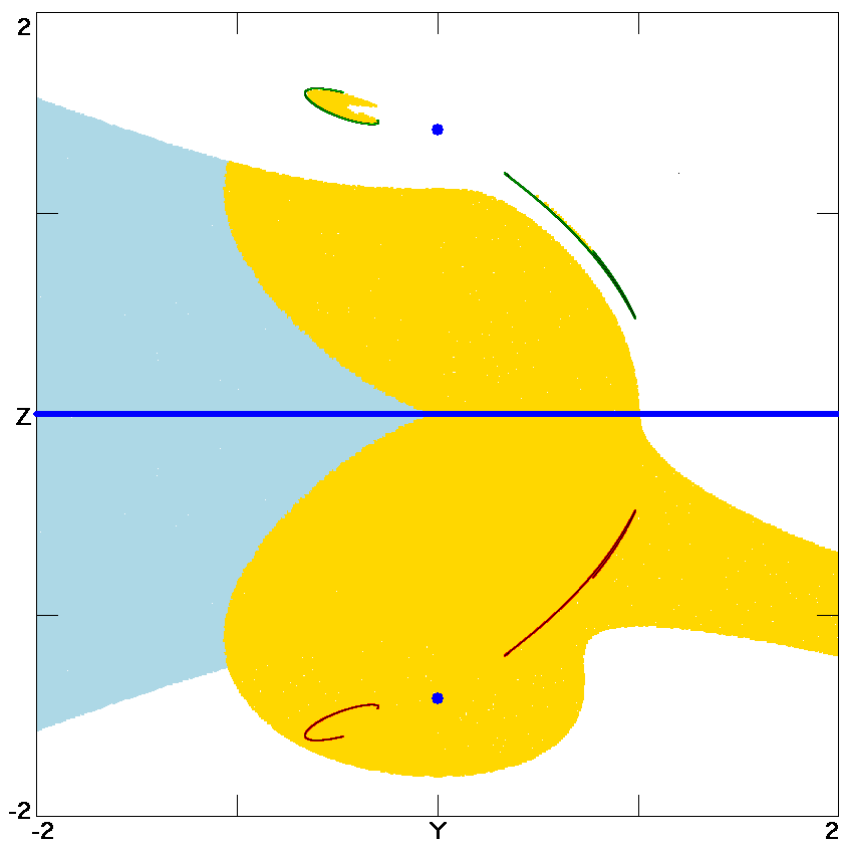

Fig. 8. Cross-section in the plane $x=-1$ of the strange attractor (red) and its symmetric repellor (green) from Eq. (13) with $a=2$. The light blue background shows initial conditions that give conservative solutions, the yellow background indicates the basin of attraction of the strange attractor, and the white background corresponds to unbounded orbits. The two point equilibria are shown as blue dots, and the line equilibrium that lies in the plane is shown as a horizontal blue line. and $( \pm 1, y, 0)$. The equilibrium points at $( \pm 1, y, 0)$ have eigenvalues $(0, \pm \sqrt{2 y})$, and the equilibrium at $(0,0, z)$ has eigenvalues that satisfy $\lambda^{3}-z \lambda^{2}+$ $a z^{2} \lambda-3 z^{3}=0$, whose solution is a spiral saddle for all $z \neq 0$.

This system also has a range of initial conditions for which the solutions are symmetric and conservative, a cross-section of which in the plane $z=-1$ is shown in Fig. 8. Note that the conservative region is symmetric about $z=0$ but the basin of attraction of the strange attractor is not symmetric. However, the solutions in the conservative region are not nested tori, but they are a dense sea of symmetric invariant periodic orbits, a few of which are shown in Fig. 9 along with the strange attractor. The periodic orbits have Lyapunov exponents $(0,0,0)$. The evidence that they are periodic as opposed to quasiperiodic is that an orbit with an initial condition such as $(-1,-2,1)$ returns repeatedly to that value within an uncertainty of $1 \times 10^{-4}$ for at least ten million cycles, which also provides confidence that the fourth-order Runge-Kutta integrator with adaptive step size is sufficiently accurate and does not introduce significant numerical damping.

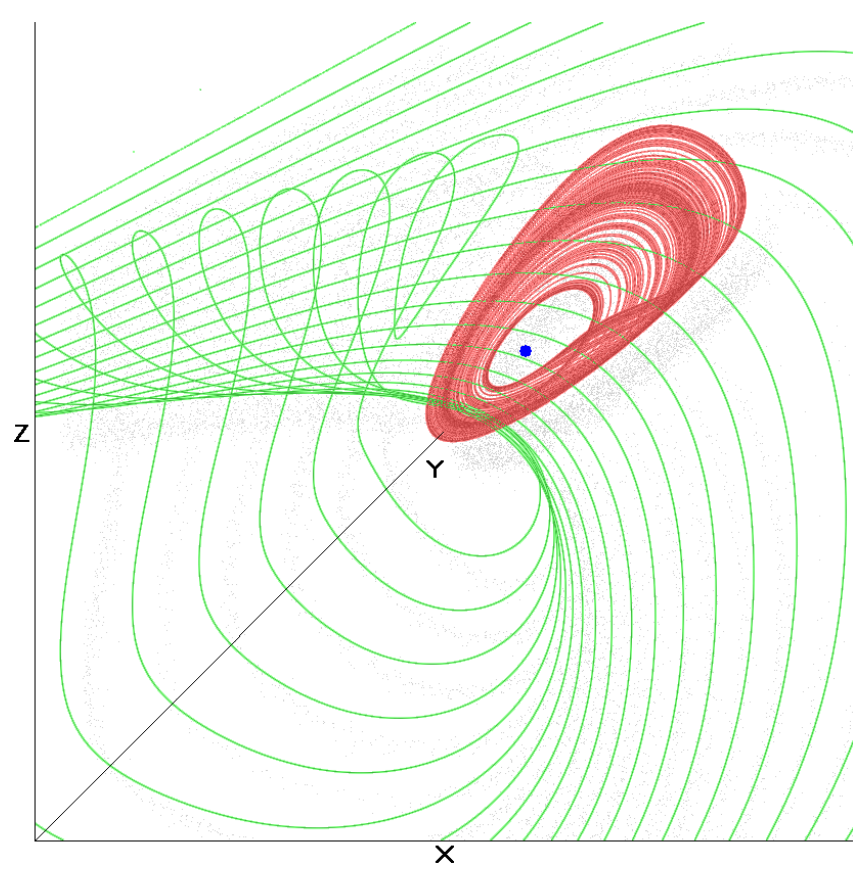

Fig. 9. A perspective view of the strange attractor (red) and a few selected conservative periodic orbits (green) for Eq. (13) with $a=2$. The point equilibrium at $(-1,0,-\sqrt{2})$ with eigenvalues $(-2.090161,0.337974 \pm 2.301872 i)$ is shown by a blue dot. 


\section{Conclusion}

Systems with all three types of involutional symmetries allow solutions that are dissipative and time-reversible. The resulting strange attractors are asymmetric but are accompanied by a twin strange repellor. Some of the attractors are hidden in the sense that their basins of attraction do not intersect small neighborhoods of any equilibrium points. Some of the systems have regions in the space of initial conditions where symmetric conservative orbits coexist with the strange attractor. These orbits are typically quasiperiodic and lie on a set of nested tori, but they can also form a dense periodic sea. This work has shown what may be the simplest examples of systems with these unusual and counterintuitive properties.

\section{Acknowledgment}

I am grateful to Chunbiao Li and Bill Hoover for helpful discussions.

\section{References}

Hoover, W. G. [1995] "Remark on 'some simple chaotic flows'," Phys. Rev. E 51, 759-760.

Hoover, W. G., Kum, O. \& Posch, H. A. [1996] "Timereversible dissipative ergodic maps," Phys. Rev. E53, 2123-2129.
Leonov, G. A., Kuznetsov, N. V. \& Vagaitsev, V. I. [2011] "Localization of hidden Chua's attractors," Phys. Lett. A 375, 2230-2233.

Leonov, G. A. \& Kuznetsov, N. V. [2013] "Hidden attractors in dynamical systems, from hidden oscillations in Hilbert-Kolmogorov, Aizerman, and Kalman problems to hidden chaotic attractor in Chua circuits," Int. J. Bifurcation and Chaos 23, 1330002-1-69.

Li, C. \& Sprott, J. C. [2014] "Chaotic flows with a single nonquadratic term," Phys. Lett. A 378, 178-183.

Moran, B., Hoover, W. G. \& Bestiale, S. [1987] "Diffusion in a periodic Lorentz gas," J. Stat. Phys. 48, 709-726.

Nosé, S. [1991] "Constant temperature molecular dynamics methods," Prog. Theor. Phys. Suppl. 103, $1-46$.

Sprott, J. C. [1994] "Some simple chaotic flows," Phys. Rev. E 50, R647-R650.

Sprott, J. C. [2014] "A dynamical system with a strange attractor and invariant tori," Phys. Lett. A 378, 1361-1363.

Sprott, J. C., Hoover, W. G. \& Hoover, C. G. [2014] "Heat conduction, and the lack thereof, in timereversible dynamical systems: Generalized NoséHoover oscillators with a temperature gradient," Phys. Rev. E 89, 042914-1-6. 